\section{OC-124 CENTRALISATION OF OG SERVICES IN THE WEST OF SCOTLAND: ARE WE MATCHING THE OUTCOMES OF THE UK NATIONAL OG AUDIT?}

doi:10.1136/gutjnl-2012-302514a.124

A Crumley, ${ }^{*}$ G Bryce, G Fullarton, C MacKay, C Craig, M Forshaw. Regional OG Unit, Glasgow Royal Infirmary, Glasgow, UK

Introduction The SAGOC report (1997-2000) highlighted the high morbidity and mortality of oesophageal and gastric resections in Scotland. Since then, centralisation into higher volume units has only slowly occurred in Scotland. The UK National OesophagoGastric Audit has not included data from Scotland and direct comparisons of outcomes have been lacking. The aim of this study was to assess the surgical outcomes following the establishment of a regional unit in 2007 relative to the outcomes of the National Oesophago-Gastric Audit 2010

Methods A prospective database (August 2007-December 2011) recorded the demographic details, treatment received, postoperative events and pathology outcomes for all patients undergoing oesophageal or gastric resections. The data were recorded into a Microsoft Excel database and wherever possible the same definitions were utilised as per the National Oesophago-Gastric Audit.

Results 233 patients (males $=174$ (75\%); median age $=66$ years) underwent either oesophageal or gastric resections, predominantly for adenocarcinoma (88\%). $67 \%$ of patients received preoperative chemotherapy. 125 patients underwent oesophagectomy: transthoracic $(n=73)$, transhiatal $(n=46)$, inoperable $(n=6) .102$ patients underwent gastrectomy: total $(n=41)$, subtotal $(n=49)$, inoperable/ bypass $(n=12)$. Six patients underwent wedge resections. 30 day and in-hospital mortality following oesophagectomy was $1.7 \%$ and $3.4 \%$ respectively, all in patients undergoing transthoracic oesophagectomy and most commonly due to respiratory complications. 30 day and in-hospital mortality following gastrectomy was 3.3\% and $5.6 \%$ respectively, all in patients undergoing total gastrectomy and most commonly due to surgical complications. 33 patients (14.1\%) were admitted to ICU most commonly for respiratory failure and following anastomotic leakage. 37 patients $(17.8 \%)$ underwent reoperation including endoscopic interventions. Clinical and radiological anastomotic leaks were observed in $12 \%$ of oesophagectomies and $10 \%$ of gastrectomies. Longitudinal and circumferential margin involvement occurred in $2.6 \%$ and $26.3 \%$ of oesophageal resections respectively. Longitudinal margin involvement was seen in $11 \%$ of gastric resections. Median lymph node was 16 (range $=3-54$ ) for all resections. More than six lymph nodes were removed in all oesophageal resections. However, $<25$ lymph nodes were removed in nearly $80 \%$ of gastric resections.

Conclusion The results from this newly established regional unit compare favourably with the outcomes from National OesophagoGastric Audit. Significant differences were highlighted in the proportion of transhiatal resections, the absence of minimally invasive surgery and longitudinal margin involvement and lymph node yields for gastric cancer surgery.

Competing interests None declared.

\section{OC-125 IRON DEFICIENCY ANAEMIA IS A COMMON PRESENTING ISSUE WITH GIANT PARAESOPHAGEAL HERNIA AND RESOLVES FOLLOWING REPAIR}

doi:10.1136/gutjnl-2012-302514a.125

S R Markar, ${ }^{*}$ P Carrott Jr, J Hong, D Low. Esophageal Surgery, Virginia Mason Medical Center, Seattle, USA, Seattle, USA

Introduction Giant Paraesophageal hiatal hernias (PEH) are most commonly associated with symptoms of chest pain, early satiety and GERD. However, Iron-deficiency anaemia is an under-appreciated condition associated with giant $\mathrm{PEH}$. The aim of this study was to evaluate the incidence of iron-deficiency anaemia in a cohort of patients with giant $\mathrm{PEH}$ and assess the incidence of resolution associated with operative PEH repair.

Methods Between 2000 and 2010, 270 patients underwent operative repair of $\mathrm{PEH}$ and were prospectively entered into an IRB-approved database. From this cohort, $123(45.6 \%)$ patients demonstrated a pre-existing diagnosis of iron-deficiency anaemia. 77 patients had a documented pre-operative haemoglobin level $(\mathrm{Hb})$ consistent with iron-deficiency anaemia and a follow-up $\mathrm{Hb}$ level at least 3 months following surgery and constituted the study population.

Results From the cohort of 77 patients with documented pre-operative iron-deficiency anaemia, 72 (94\%) underwent elective PEH repair, with a median age was 75 (range 39-91). Cameron erosions were endoscopically documented preoperatively in 25 patients (32\%). The average preoperative $\mathrm{Hb}$ value was 11.8 (7.6-16). Postoperatively at 3-12 month follow-up, the average $\mathrm{Hb}$ level was 13.2 (10.7-17), and at more than 1 year follow-up it was 13.6 (9.2-17.2) $(p<0.05)$. Furthermore $90 \%$ of patients had a rise in post-operative haemoglobin level by at least $1 \mathrm{~g} / \mathrm{dl}$. Anaemia was fully resolved postoperatively ( $\mathrm{Hb} \geq 12.0$ in females, $\geq 14.0$ in males) in $55(71 \%)$ patients. This resolution was observed more commonly in women $(40 / 50,80 \%)$ than men $(15 / 27,56 \%, \mathrm{p}<0.05)$. Also younger patients ( $<70$ years) were more likely to resolve their anaemia (29/33 vs 26/ 44; $\mathrm{p}<0.05)$ and have a greater post-operative $\mathrm{Hb}(14.0 \mathrm{vs} 13.0 \mathrm{~g} / \mathrm{dl}$; $\mathrm{p}<0.05)$ than older patients. 40 patients required preoperative iron supplementation, 29 (73\%) were able to discontinue iron following surgery. There was no significant difference in the resolution of anaemia in patients with or without Cameron erosions (19/25 vs $36 / 52, p=0.54)$.

Conclusion This single institution study shows a high incidence of iron-deficiency anaemia (45.6\%) in patients with giant PEH. Elective repair results in resolution of the anaemia and discontinuation of iron supplementation therapy, in more than $70 \%$ of patients. This improvement in $\mathrm{Hb}$ is independent of the presence of pre-operative Cameron erosions. This study demonstrates the clinical and potential economic benefits of elective PEH repair of patients with Giant PEH and iron-deficiency anaemia.

Competing interests None declared.

\section{OC-126 TRANSLOCATION OF MULTIDISCIPLINARY STANDARDISED CLINICAL PATHWAYS BETWEEN HOSPITALS AND HEALTH SYSTEMS IS FEASIBLE AND CAN PRODUCE IMMEDIATE IMPROVEMENT IN PERIOPERATIVE OUTCOMES OF SURGICAL TREATMENT OF OESOPHAGEAL CANCER}

doi:10.1136/gutjnl-2012-302514a.126

${ }^{1} \mathrm{~S}$ Preston, ${ }^{*}{ }^{2} \mathrm{~S} R$ Markar, ${ }^{1} \mathrm{C}$ Baker, ${ }^{1} Y$ Soon, ${ }^{1} \mathrm{~S}$ Singh, ${ }^{2} \mathrm{D}$ Low. ${ }^{1}$ Royal Surrey County Hospital, Guildford, UK; ${ }^{2}$ Virginia Mason Medical Center, Seattle, USA, Seattle, USA

Introduction Standardised postoperative care pathways (SPCP) allow the introduction of a targeted goal-directed approach to recovery following major cancer surgery. A standardised oesophagectomy clinical pathway was established at Virginia Mason Medical Center (VMMC), Seattle, USA in 1991 and has undergone five revisions up to 2011. No information exists as to whether pathways can be successfully relocated between different cancer programs and health systems. In March 2011 a multidiscliplinary team from the Royal Surrey County Hospital (RSC) visited VMMC and instituted a similar pathway in April 2011. The aim of this study is to determine the effect of the translocation and implementation of an oesophagectomy care pathway on postoperative outcomes within a 1 year time period. 
Abstract 0C-126 Table 1 Results

\begin{tabular}{|c|c|c|c|c|c|}
\hline Outcome & VMMC (1) & Prepathway (2) & Nonpathway (3) & Pathway (4) & p Value \\
\hline Pts 1st day mobilisation (\%) & 93 & 8.3 & 42 & 100 & $\begin{array}{l}>0.99(1 \text { vs } 4) \\
<0.05 \text { (2 vs } 4) \\
0.16 \text { (2 vs } 3)\end{array}$ \\
\hline Complications (\%) & 47.3 & 75 & 75 & 33.3 & $\begin{array}{l}0.53 \text { (1 vs } 4) \\
<0.05 \text { (2 vs } 4)\end{array}$ \\
\hline ICU stay (days) & $1(0-22)$ & $4(2-20)$ & $3(2-9)$ & $3(1-5)$ & $\begin{array}{l}<0.05 \text { ( } 1 \text { vs } 4,2 \text { vs } 4) \\
0.13 \text { ( } 2 \text { vs } 3)\end{array}$ \\
\hline Hospital stay (days) & $8(6-54)$ & $17(12-30)$ & $13(8-22)$ & $7(6-37)$ & $\begin{array}{l}0.25 \text { (1 vs } 4) \\
<0.05 \text { (2 vs } 4) \\
0.09 \text { (2 vs } 3 \text { ) }\end{array}$ \\
\hline
\end{tabular}

Methods Four groups were included in this study:

1. 74 patients operated on at VMMC (2009-2011).

2. 12 RSC patients operated on before the introduction of SPCP.

3. 12 RSC patients operated on after the introduction of SPCP but not included.

4. 12 RSC patients managed according to the SPCP.

Groups were compared with respect to patient demographics, peri- and post-operative outcomes.

Results Presenting demographics were similar in all groups with respect to BMI, medical co-morbidities, ASA grade and clinical stage. Age was significantly reduced Group 4 compared to Group 2 $(\mathrm{p}<0.05)$. Following initiating SPCP the use of feeding jejunostomies increased $(67 \%-100 \% ; \mathrm{p}<0.05)$ as did immediate extubation $(67 \%-$ $100 \% ; p<0.05)$. Further statistically significant improvements were noted in complications, length of ICU and hospital stay. Group 3 also showed a less significant improvement in length of ICU and hospital stay, and first day mobilisation.

Conclusion The results of this study demonstrate the potential for accelerated improvement in short-term outcomes following oesophagectomy through the translocation and integration of standardised postoperative pathways. SPCP can also change hospital processes to improve outcomes in other patients. A multidisciplinary approach with involvement of the entire health care team in the design, implementation, evaluation and revision of these pathways is essential and will help ensure all team members are committed to achieving specific targeted pathway goals.

Competing interests None declared.

\section{OC-127 ONE THOUSAND LAPAROSCOPIC ROUX-EN-Y GASTRIC BYPASSES: PERIOPERATIVE AND LONG TERM OUTCOMES}

doi:10.1136/gutjnl-2012-302514a.127

J C Tham, * D Pournaras, I Finlay, D Titcomb, J Edmond, D Mahon, R Welbourn. Musgrove Park Hospital, Taunton, UK

Introduction Laparoscopic Roux-en-y gastric bypass (LRYGB) has been established as an effective treatment for obesity and obesity related morbidity. However, safety of LRYGB remains under public scrutiny. Defining operative outcomes is vital to establish its safety and long term efficacy.

Methods One thousand consecutive patients underwent LRYGB as a primary procedure by two surgeons in a single centre from 2004 to 2011. Twenty-six patients had intra-gastric balloon insertion before LRYGB to reduce Body Mass Index (BMI). LRYGB was done using a retrocolic, antegastric linear staple technique with routine closure of internal hernia defects. Patient demographics and complications were collected prospectively in a local database, and after January 2009, in the National Bariatric Surgery Registry. The Obesity Surgery Mortality Risk Score (OS-MRS) was used for risk stratification. Analysis was performed on each 100 patients making up 10 groups sequentially with the first 100 in group 1.
Results Mean age \pm SD was $45.5 \pm 10.4$ with mean BMI of $49.5 \pm 7.7$ and $79.3 \%$ were female. There were 61 (6.1\%) high-risk patients (OS-MRS class C). The follow-up rate up to 6 years was $87.9 \%$. There were four conversions-to-open surgery in the first 400 patients with no conversions thereafter. Mean \% excess weight loss (EWL) pre-operatively as compared to initial weight was $11.6 \% \pm$ 12.5. \% EWL subsequently was: 1 year $67.7 \% \pm 18.9 ; 2$ years: $71.6 \% \pm$ 20.3; 5 years: $57.5 \% \pm 21.8$; 6 years: $61.1 \% \pm 21.4$. Overall, the 30 -day complication rate was $5.5 \%$ with a 30 -day reoperation rate of $2.9 \%$. The 30-day complication rate fell over time; 13 patients had a complication in group 1 compared to 1 patient in group $10(p=0.03)$. Further, there was significant reduction in complications between groups 1 and $2(p<0.001)$ but no difference between each group thereafter. Major complications within 30 days postoperatively were gastrointestinal leak $(0.6 \%)$, gastro-jejunal stenosis $(0.3 \%)$, stenosis at the mesocolic defect $(0.3 \%)$, jejuno-jejunostomy hernia $(0.7 \%)$, mesocolic hernia (1\%) and bleeding $(2.1 \%)$. There was one death $(0.1 \%)$ within the 30 postoperative days (in group 2$)$. The cumulative complication rate at 6 years was $9.1 \%$ with $5.8 \%$ needing re-operation or endoscopic intervention. The mean operative time per group for the first 600 patients was, in minutes, $163 \pm 53.7,129.2 \pm 109.8,100.0 \pm 27.8,104.3 \pm 28.9,92.7 \pm 32.1$ and $93.8 \pm 30.9$, a significant decrease between groups 1 and $2(p=0.003)$, 2 and $3(p=0.007)$, and 4 and $5(p=0.008)$ respectively, but not between 3 and 4 .

Conclusion LRYGB is a safe and efficacious procedure with sustained weight loss at 6 years. The learning curve is about 100 cases but operative time continues to improve up to the 500th case. A high volume centre is associated with low morbidity and mortality.

Competing interests None declared.

\section{OC-128 THE EFFECT OF OBESITY ON THE RADICALITY OF SUBTOTAL OESOPHAGECTOMY FOR OESOPHAGEAL ADENOCARCINOMA}

doi:10.1136/gutjnl-2012-302514a.128

H V Jones, ${ }^{*}$ S Wahed, A Krishnan, J Shenfine, S M Griffin. Northern OesophagoGastric Cancer Unit, Royal Victoria Infirmary, Newcastle Upon Tyne, UK

Introduction Obesity is on the increase in the UK and is a known risk factor for adenocarcinoma of the oesophagus. It is recognised that oesophagectomy in obese patients is more difficult with concerns that radicality of resection is reduced. The aims of this study were to evaluate body mass index (BMI) in patients with oesophageal adenocarcinoma who underwent subtotal oesophagectomy with radical lymphadenectomy and to evaluate the effect of obesity on lymph node (LN) dissection and survival.

Methods All patients who underwent subtotal oesophagectomy for adenocarcinoma between January 2000 and December 2010 were identified from a prospectively maintained database. All other histological types were excluded. Patients were categorised according to BMI using the WHO criteria: underweight (2), normal 